\title{
Wnt5a is associated with the differentiation of bone marrow mesenchymal stem cells in vascular calcification by connecting with different receptors
}

\author{
SIMING GUAN $^{1}$, ZHIMIN WANG $^{2}$, FANG XIN $^{1}$ and HUAPING XIN ${ }^{2}$ \\ ${ }^{1}$ Department of Geriatrics, Union Hospital, Tongji Medical College, Huazhong University of Science and Technology, Wuhan, \\ Hubei 430022; ${ }^{2}$ Department of Neurology, The First People's Hospital of Taizhou, Taizhou, Zhejiang 318020, P.R. China
}

Received December 1, 2013; Accepted May 23, 2014

DOI: $10.3892 / \mathrm{mmr} .2014 .2449$

\begin{abstract}
Vascular calcification significantly affects the health of the elderly. Increasing evidence proved that vascular calcification is an actively regulated osteogenic process. The osteochondrocytic differentiation of mesenchymal stem cells (MSCs) is a significant step of osteogenic processes. The Wnt pathways has been identified as contributing to the regulation of osteogenic mineralization during development and disease. However, it remains unknown whether these MSCs in the vascular calcification differentiate into normal vascular smooth muscle cells (VSMCs) in vivo in order to treat damaged vascular tissue or into calcified VSMCs to aggravate calcification correlated to the Wnt pathways. Thus, it is necessary to analyze the mechanisms of MSC differentiation in detail. In the present study a cell-cell co-culturing in vitro system was used to observe MSCs that directly interact with normal or calcified VSMCs during calcification and to investigate the gene expression of the Wnt pathways during the process. Direct co-cultures were established by seeding two different cell types, VSMCs or calcified VSMCs, or a mixture of both at ratios of 5,000:5,000 cells $/ 1.7 \mathrm{~cm}^{2}$ onto either gelatin-coated $1.7-\mathrm{cm}^{2}$ chamber slides for immunohistochemical analysis or gelatin-coated $75-\mathrm{cm}^{2}$ tissue culture flasks for protein or RNA isolation. Osteoblastic differentiation was evaluated by examining the cell morphology and assessing the activity of alkaline phosphatase in the cell lysates by alkaline phosphatase staining. Additionally, the mRNA expression levels of the genes encoding for proteins involved in the Wnt signaling proteins, Wnt5A, LRP6, Ror2, c-Jun-N-terminal kinase and $\beta$-catenin, were assessed in each group. The present study demonstrated that Wnts are expressed in the progress of differentiation of MSCs during calcification.
\end{abstract}

Correspondence to: Mrs Huaping Xin, Department of Neurology, The First People's Hospital of Taizhou, 218 Hengjie Road, Taizhou, Zhejiang 318020, P.R. China

E-mail: xhp691@sohu.com

Key words: vascular calcification, direct co-cultures, mesenchymal stem cells, Wnt5a, different receptors
MSCs can differentiate into different cell phenotypes when there is direct cell-cell contact with VSMCs or calcified VSMCs, and the Wnt5a/Ror2 signaling pathway may be associated with the determination of differentiation of MSCs in this process.

\section{Introduction}

Vascular calcification significantly affects the health of the elderly. Increasing evidence has indicated that vascular calcification is an actively regulated osteogenic process (1). In addition, the osteochondrocytic differentiation of bone marrow-derived mesenchymal stem cells (MSCs) is a significant step in the osteogenic processes (2). MSCs, a main population of stem cells, which reside in the bone marrow, adipose tissue, peripheral blood and synovium (3), are able to differentiate into multiple cell lineages, including osteoblasts, adipocytes, chondrocytes and cardiac myocytes (4). Additionally, they are able to differentiate into functional regenerative units (5). Furthermore, previous studies have revealed anti-inflammatory properties of MSCs, indicating their usefulness in both organ transplantation and treatment of autoimmune diseases (6).

Indeed, a number of preclinical and clinical studies in diseases, including multiple sclerosis (7), diabetes (8), osteogenesis imperfecta (9) and cartilage defect (10), have already demonstrated that there is therapeutic potential of MSCs. Successful exploitation of MSCs has been reported in several preclinical models (11) and these models have demonstrated that they enhanced tissue repair either by direct regeneration (12) or by secreting paracrine factors (8). The therapeutic ability of MSCs has been augmented by genetic modifications (5), which overexpress prosurvival, or growth factor genes $(6,9)$. In clinical trials, MSCs have been able to repair injured myocardium (13), bone (14) and soft tissue (11). Although MSC growth in injured vascular tissue may regenerate normal vascular tissue, it can also produce ectopic tissues $(10,11)$, including those observed in advanced atherosclerotic calcification. MSCs are involved in the initiation and progression of various vascular diseases (15). One cannot rule out the possibility of severe or delayed side effects that may have been observed in clinical trials $(10,11)$. However, the underlying mechanisms of the therapeutic effects of MSCs remain elusive and controversial. 
The Wnt pathways regulate endothelial dysfunction and vascular smooth muscle cell (VSMC) proliferation and migration, and thereby intimal thickening (16). Furthermore, they can regulate inflammation (17) and foam cell formation (16), pathological angiogenesis (1) and calcification (18), which are crucial processes in plaque formation and stability (16). Of note, the Wnt pathways, which have been identified as contributing to the regulation of osteogenic mineralization during development and disease (18), control the fate of MSCs (19). It remains unknown whether these MSCs during the vascular calcification differentiate into normal VSMCs in vivo to treat damaged vascular tissue or to calcified VSMCs to aggravate calcification correlated to the Wnt pathways. At present, preventing MSC pathological differentiation is a powerful and potential strategy to delay aging of individuals and to promote application of cell therapy for treating age-associated diseases (20). Therefore, it is required to analyze mechanisms of MSC differentiation in detail. In the present study the cell-cell co-culturing system was used in order to observe in vitro MSCs directly interact with normal or calcified VSMCs during calcification and to investigate the gene expression of Wnt pathways during the process.

\section{Materials and methods}

Rat bone-marrow-derived MSCs. The isolation and culturing of male MSCs were performed as previously published (21). MSCs were cultured in Dulbecco's modified Eagle's medium (DMEM), supplemented with $10 \%$ heat-inactivated fetal bovine serum (FBS), $100 \mathrm{U} / \mathrm{ml}$ penicillin and $100 \mathrm{Ag} / \mathrm{ml}$ streptomycin (all Gibco Life Technologies, Carlsbad, CA, USA) at $37^{\circ} \mathrm{C}$ in $5 \% \mathrm{CO}_{2}$ and $95 \%$ air. MSCs were used at passage three.

Rat aortic SMCs. Rat aortic VSMCs (A-10; ATCC, Manassas, VA, USA) were grown in low-glucose DMEM. Osteosynthesis-inducing medium (OS), which was used only in one osteoblastic differentiation assay, contained DMEM with $0.1 \mu \mathrm{M}$ dexamethasone, $10 \mathrm{mM}$ sodium $\beta$-glycerol-phosphate and $0.05 \mathrm{mM}$ ascorbic acid-2-phosphate (Sigma-Aldrich, St. Louis, MO, USA). The culture medium was removed and replaced with fresh medium three times a week. VSMCs were cultured for 21 days following the formation of calcified nodules.

Co-culture conditions. Direct co-cultures were established by seeding two different cell types: VSMCs or calcified VSMCs and MSCs together at a ratio of 5,000:5,000 cells/1.7 $\mathrm{cm}^{2}$ onto either gelatin-coated $1.7-\mathrm{cm}^{2}$ chamber slides (Becton-Dickinson, Oxford, UK) for immunohistochemical analysis, or gelatin-coated $75-\mathrm{cm}^{2}$ tissue culture flasks (Becton-Dickinson) for protein or RNA isolation. The cells were cultured in a 50:50 mix of growth medium for the two cell types used at $37^{\circ} \mathrm{C}$ in a humidified atmosphere of $5 \% \mathrm{CO}_{2}$ in air, with the medium replaced after $24 \mathrm{~h}$. Next, the cells were cultured in a low-glucose DMEM supplemented with $10 \%$ FBS at $37^{\circ} \mathrm{C}$ in a $5 \% \mathrm{CO}_{2}$ incubator for 14 days. The medium was changed every three days. Each co-culture experiment was performed three times in order to validate the results. The 8 groups were as follows: GS (calcified SMCs); GS+M (calcified SMCs co-cultured with MSCs); GS+M+OS (MSCs and calcified SMCs cultured in OS);
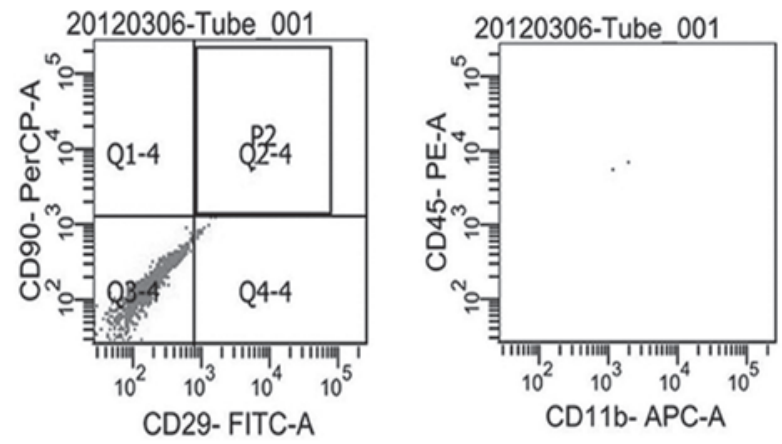

Figure 1. MSCs cell-surface antigen analysis. The levels of CD29 and CD90 expression were high (95\%), while those of CD45 and CD11b were low $(0.7 \%)$. MSCs, mesenchymal stem cells; FITC, fluorescein isothiocyanate; PE, phycoerythrin; PerCP, peridinin chlorophyll protein; APC, allophycocyanin.

S (SMCs); S+M (MSCs co-cultured with SMCs); S+M+OS (SMCs and MSCs cultured in OS); M (MSCs); M+OS (MSCs cultured in OS)

Osteoblastic differentiation was evaluated by the cell morphology and activity of alkaline phosphatase (22) in cell lysates and alkaline phosphatase (ALP) staining. Additionally, by investigating the mRNA expression levels of genes encoding for proteins involved in Wnt signaling, Wnt5a, LRP6, Ror2, c-Jun-N-terminal kinase (JNK) and $\beta$-catenin in each group.

Flow cytometry. In analogy with a previous study (21), passage 3 MSCs were trypsinized, washed with phosphate-buffered saline (PBS) and incubated with fluorescein isothiocyanate phycoerythrin-conjugated monoclonal antibodies (Biolegend, San Diego, CA, USA) specific against CD29, CD90, CD45 or CD11b, or with $\mathrm{PBS}$ at $4^{\circ} \mathrm{C}$ for $30 \mathrm{~min}$. The analysis was performed by flow cytometry (Becton-Dickinson, Franklin Lakes, NJ, USA) using Cell Quest software (Becton-Dickinson).

von Kossa staining. For von Kossa staining, cells were fixed at $4^{\circ} \mathrm{C}$ for $45 \mathrm{~min}$ with paraformaldehyde (Sigma-Aldrich), then the fixed cells were incubated in 5\% silver nitrate for $30 \mathrm{~min}$ under ultraviolet light and air-dried until the development of a black color. Calcification was observed under a CX31 light microscope (Olympus Corporation, Tokyo, Japan).

$A L P$. ALP activity and expression were assessed as previously described (21). The cells were fixed with $4 \%$ paraformaldehyde (Sigma-Aldrich) and then stained with the 5-bromo-4-chloro-3'-indolyphosphate and nitro-blue tetrazolium (BCIP/NBT) phosphatase substrate system (KLP, Gaithersburg, MD, USA) following the manufacturer's instructions. The ALP activity was determined in all the samples. The samples were extracted with an assay buffer containing $50 \mathrm{mM}$ Tris- $\mathrm{HCl}, 0.1 \%$ Triton $\mathrm{X}-100$ and $0.9 \% \mathrm{NaCl}(\mathrm{pH} 7.6)$, and the lysate was frozen. Lysate samples were then thawed and the enzyme activity was determined in duplicates using 0.1 M 4-p-nitrophenylphosphate as a substrate (Nanjing Jiancheng Bioengineering Institute, Nanjing, China). The absorbance was measured at $492 \mathrm{~nm}$ using a iMark Microplate Absorbance Reader (Bio-Rad, Hercules, CA, USA). The total 


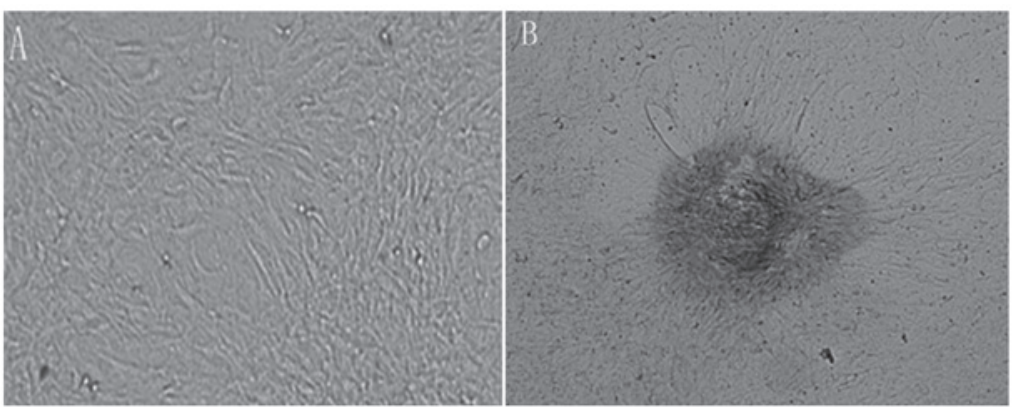

Figure 2. Von Kossa staining: (A) Normal and (B, black arrowhead) calcified vascular smooth muscle cells. Original magnification, x100.

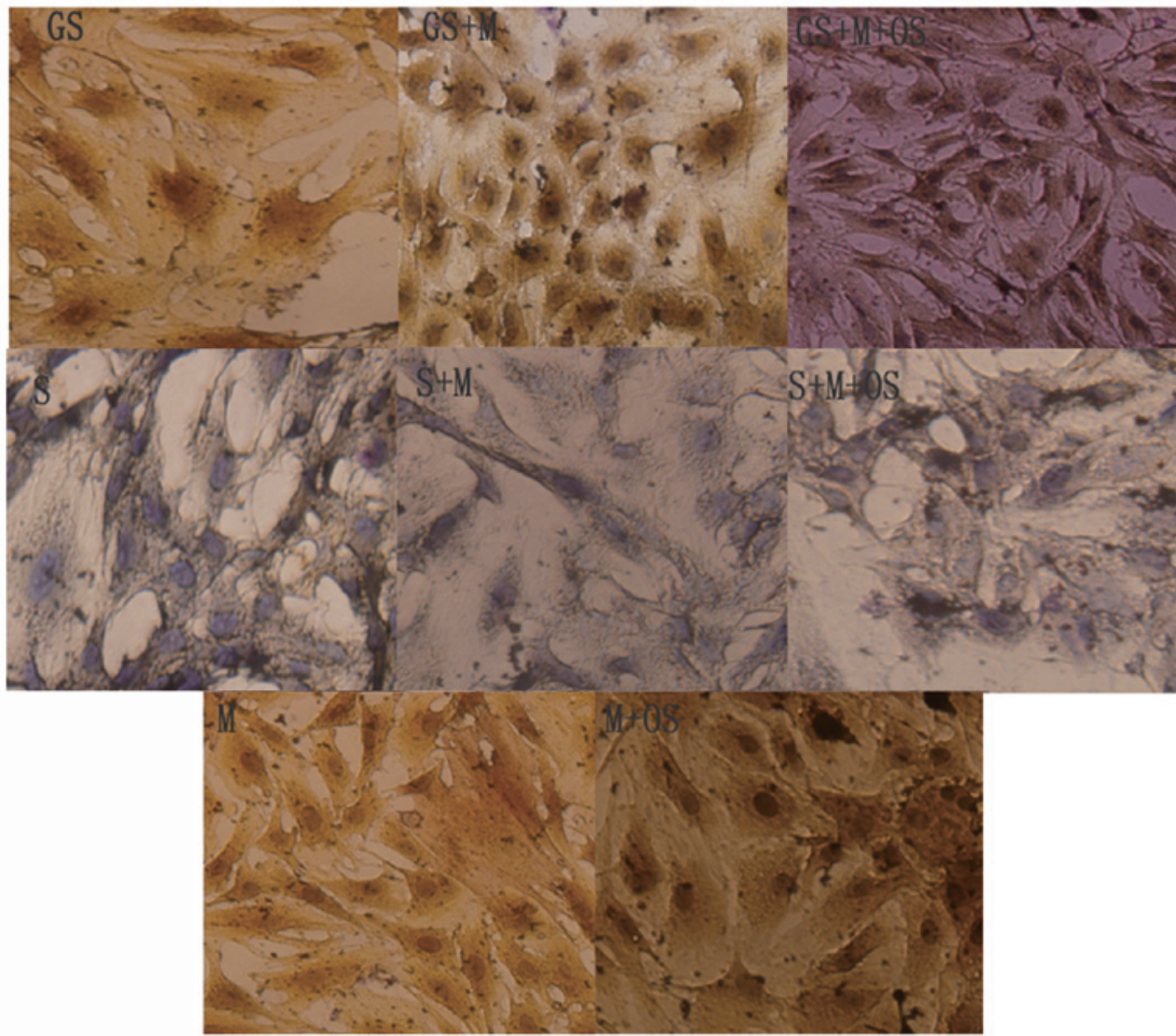

Figure 3. ALP staining (original magnification, $\mathrm{x} 400$ ): Staining was positive when MSCs were directly co-cultured with calcified VSMCs, which was most significant in the GS+M+OS group. However, ALP staining was negative when cells were co-cultured with non-calcified VSMCs. There was no significance compared with VSMCs cultured alone. The 8 groups were as follows: GS (calcified SMCs); GS+M (calcified SMCs co-cultured with MSCs); GS+M+OS (MSCs and calcified SMCs cultured in OS); S (SMCs); S+M (MSCs co-cultured with SMCs); S+M+OS (SMCs and MSCs cultured in OS); M (MSCs); M+OS (MSCs cultured in OS) ALP, alkaline phosphatase; MSCs, mesechymal stem cells; VSMCs, vascular smooth muscle cells; OS, osteosynthesis-inducing medium.

protein contents were determined by the Bio-Rad protein assay (Bio-Rad).

Expression of genes encoding Wnt-signaling proteins. The total RNA was isolated from the samples using TRIzol reagent according to the manufacturer's instructions (Invitrogen, Carlsbad, CA, USA) and reverse-transcribed into cDNA with a reverse transcription kit (Toyobo, Osaka, Japan). Quantitative polymerase chain reaction (qPCR) was performed with an $\mathrm{ABI}$ PRISM 7900 sequence detector system (Applied Biosystems) according to the manufacturer's instructions. Actin was used as an endogenous control. The PCR reaction mixture contained SYBR green I (Takara), cDNA and the primers. The following sequences of primers for qPCR were used: Actin (110-bp), forward 5'-CGTTGACAT-CCGTAAAGACCTC-3' and reverse 5'-TAGGAGCCAGGGCAGTAATCT-3'; $\beta$-catenin (190-bp), forward 5'-GGTGAAAATGCTTGGGTCG-3' and reverse 5'-GATCTGAAGGCAGTCTGTCGTA-3'; JNK (132-bp), forward 5'-AGCAGT-AGCCCCGCCAGTA-3' and reverse 5'-TGTCAGTGTCCTTCCCACCTC-3'; Ror2 (154-bp), forward 5'-TGGGAACCGAACTATTTATGTG-3' and reverse 5'-AGGAAAGACGAAGTGGCAGA-3'; Wnt5a (236-bp), forward 5'-CAACAG-CCGCTTCAACTCC-3' and reverse 5'-TGACATAGCAGCACCAGTGA-3'; and LRP6 (231-bp), forward 5'-TGATAACCAGTTCACGGATGC-3' and reverse 5'-CCATTTGACAGGCGGAAAG-3'. All experiments were 
A

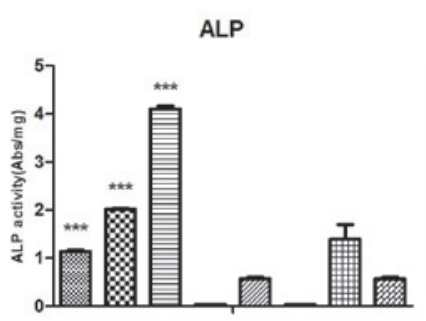

$\mathbf{C}$

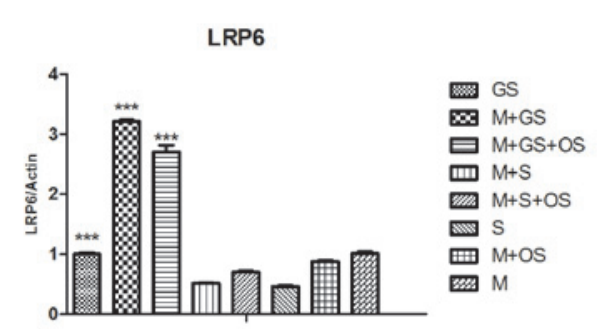

$\mathbf{E}$

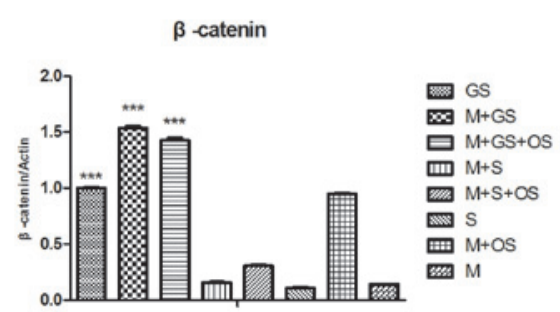

B

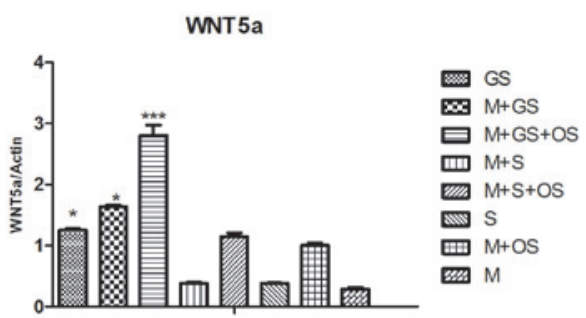

D

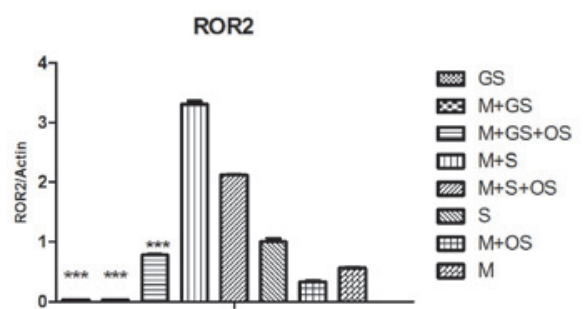

$\mathbf{F}$

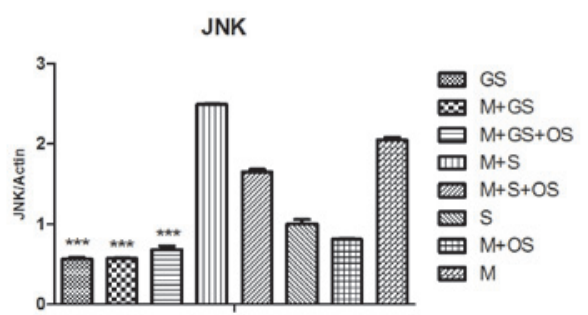

Figure 4. Direct co-cultures were established by two different cell types, normal or calcified SMCs, being mixed with MSCs at a ratio of 5,000:5,000. Next, cells were cultured for 14 days and the mRNA expression levels of the genes encoding Wnt-signaling proteins, Wnt5a, LRP6, Ror2, JNK and $\beta$-catenin, were investigated. (A) ALP activity of each groups. (B) mRNA levels of wnt5a. (C) mRNA levels of LRP6. (D) mRNA levels of Ror2. (E) mRNA levels of $\beta$-catenin. (F) mRNA levels of JNK. ${ }^{* * *} \mathrm{P}<0.01,{ }^{*} \mathrm{P}<0.05$, compared with the $\mathrm{M}+\mathrm{S}$ group, the $\mathrm{M}+\mathrm{S}+\mathrm{OS}$ group, the $\mathrm{S}$ group. The 8 groups were as follows: GS (calcified SMCs); GS+M (calcified SMCs co-cultured with MSCs); GS+M+OS (MSCs and calcified SMCs cultured in OS); S (SMCs); S+M (MSCs co-cultured with SMCs); S+M+OS (SMCs and MSCs cultured in OS); M (MSCs); M+OS (MSCs cultured in OS) SMCs, smooth muscle cells; MSCs, mesenchymal stem cells; JNK, c-Jun-N-terminal kinase; ALP, alkaline phosphatase; OS, osteosynthesis-inducing medium.

performed in duplicate and normalized to actin as an invariant endogenous control. The PCR conditions were $50^{\circ} \mathrm{C}$ for $2 \mathrm{~min}$ and $94^{\circ} \mathrm{C}$ for $2 \mathrm{~min}$, followed by 40 cycles of $94^{\circ} \mathrm{C}$ for $15 \mathrm{sec}$ and $60^{\circ} \mathrm{C}$ for $30 \mathrm{sec}$. The results were calculated using the $\Delta \Delta \mathrm{CT}$ method and are presented as the fold increase relative to actin expression.

Statistical analysis. Values are presented as the mean \pm standard error of the mean. The significance of differences was estimated by analysis of variance followed by Student-Newmann-Keuls multiple comparison tests. $\mathrm{P}<0.05$ was considered to indicate a statistically significant difference between values. All statistical analyses were performed using SPSS software (version 11.0; SPSS Inc. Chicago, IL, USA).

\section{Results}

Cultured passage three MSCs. Passage three rat MSCs were positive for CD29 and CD90, while they were negative for CD45 and CD11b, and cells were different from hemopoietic stem cells (Fig. 1).

Calcified VSMCs. VSMCs were cultured in OS for 21 days. Representative images of von Kossa staining are shown in Fig. 2. Von Kossa staining revealed that no calcification was present in
VSMCs cultured without OS (Fig. 2A), and there were evidently positively-stained, black, calcified nodules in VSMCs cultured with OS (Fig. 2B, black arrows).

MSCs cultured in OS did not exhibit an osteoblast phenotype when in direct contact with non-calcified VSMCs. As previously published by our group (21), OS did not increase the ALP activity when MSCs were cultured with non-calcified VSMCs. ALP staining was negative (Fig. 3), and there were no differences in ALP activity between the S and S+M groups; however, ALP activity was slightly elevated in the $\mathrm{S}+\mathrm{M}+\mathrm{OS}$ group $(\mathrm{P}<0.05)$.

MSCs exhibit an osteoblast phenotype when in direct contact with calcified VSMCs. ALP staining was positive when MSCs were directly co-cultured with calcified VSMCs, which was most significant in the GS+M+OS group. However, ALP staining was negative when MSCs were directly co-cultured with non-calcified VSMCs, and there was no significant difference compared with that of VSMCs cultured alone (Fig. 3). In addition, ALP activity was more significant in the GS+M+OS and GS+M groups compared with that in the GS group $\left({ }^{* * *} \mathrm{P}<0.01\right.$; Fig. 4A).

Different expression levels of genes encoding for proteins involved in Wnt signaling may be associated with the differentiation of MSCs cultured with normal or calcified VSMCs. 
In order to directly investigate the changes in the expression of proteins involved in the Wnt pathway, the gene expression levels of Wnt5a, the Wnt co-receptors LRP6 and Ror2, as well as the Wnt production of JNK and $\beta$-catenin were assessed in each group. Wnt5a levels were highest in the M+GS, M+GS+OS, GS and M+OS groups $\left({ }^{* * *} \mathrm{P}<0.001\right)$; however, they were low in the $\mathrm{M}+\mathrm{S}$ and $\mathrm{S}$ groups $\left({ }^{* * *} \mathrm{P}<0.001\right)$ (Fig. 4B). The gene expression levels of LRP6 and $\beta$-catenin were significantly reduced in the $\mathrm{M}+\mathrm{S}, \mathrm{M}+\mathrm{S}+\mathrm{OS}$ and $\mathrm{S}$ groups; whereas they were increased in the M+GS, M+GS+OS, GS and M+OS groups $\left({ }^{* * *} \mathrm{P}<0.001\right.$; Fig. 4C and E). Of note, the mRNA levels of Ror2 and JNK were markedly reduced in the M+GS, M+GS+OS, GS and M+OS groups only, while they were increased in the $\mathrm{M}+\mathrm{S}, \mathrm{M}+\mathrm{S}+\mathrm{OS}, \mathrm{S}$ and $\mathrm{M}$ groups $\left({ }^{* * *} \mathrm{P}<0.01\right.$; Fig. 4D and $\left.\mathrm{F}\right)$. Although decreases in Ror2 and JNK expression were also apparent in the M+GS, M+GS+OS, GS and M+OS groups, they were not statistically significant $(\mathrm{P}>0.05)$. No differences were identified between the mRNA expression levels of the LRP6 and $\beta$-catenin genes investigated in the $\mathrm{M}+\mathrm{S}, \mathrm{M}+\mathrm{S}+\mathrm{OS}$ and $\mathrm{S}$ groups $(\mathrm{P}>0.05)$.

\section{Discussion}

MSCs have a critical role in tissue regeneration and homeostasis. However, it is noteworthy that the present study has identified that circulating concentrations of stem cell-mobilizing cytokines were associated with the levels of osteoprogenitor cells and aortic calcification severity (23). The major factors, resident cellular $(15,23)$ and local environment (24), are likely to determine the fate of MSCs. Additionally, previous studies indicated that direct cell-to-cell contact between resident cells and MSCs was critical in the differentiation of MSCs (25). The present study examined MSCs co-cultured with non-calcified or calcified VSMCs in vitro by a direct cell-cell co-culturing system. The data of the present study indicated that direct cell-to-cell contact between resident cells, VSMCs and MSCs, was decisional in the differentiation of MSCs into non-calcified or calcified VSMCs.

Numerous cell surface receptors have been used to create functional surfaces in order to enhance cell adhesion or to alter cell morphology, including cell adhesion molecules (26), cadherins (27), etc. Wnt-signaling provides instructive cues for the recruitment, maintenance and differentiation of MSCs (28), which is mediated through cell-cell interactions and is involved in numerous developmental processes and cellular functions. Wnt signaling has been implicated in the self-renewal and maintenance of pluripotent stem cells $(7,29)$. Wnts are a family of secreted glycoproteins that bind to a class of Frizzled (Frz) receptors (30). The conserved Wnt cascade was composed by the canonical Wnt/ $\beta$-catenin and non-canonical Wnt/Ror2 pathways. It has been reported that canonical Wnt-signaling can activate osteogenesis in mineralization in certain cellular contexts, and promotes the osteoblastogenesis of murine pluripotent mesenchymal and osteoprogenitor cells by upregulation of runt-related transcription factor 2 or osterix (31). Additionally, $\beta$-catenin is a transcriptional co-adaptor that is indispensable for osteogenic tissue mineralization and takes part in osteochondrogenic differentiation of mural mesenchymal progenitors (32).
Stabilization of cytoplasmic $\beta$-catenin is the hallmark of activated canonical Wnt signaling $(33,34)$. Mice with stabilized $\beta$-catenin expressed in their cardiomyocytes also revealed a functional decline following injury (33). Therefore, numerous and diverse studies have converged on $\beta$-catenin activation as a key component of arteriosclerotic physiology.

The low-density lipoprotein (LDL) receptor-related protein (LRP) family is well known for its ability to bind multiple ligands, including apolipoprotein E, very LDL remnants, lipoprotein lipase, tissue-type and urokinase-type plasminogen activators and thrombospondin I. To date, studies have only identified LRP5 and LRP6 as coreceptors for the canonical Wnt signaling cascade. LRP6 is a single-pass transmembrane protein composed of four extracellular epidermal growth factor type repeats and three LDLR repeats (28). The important role of the cytoplasmic region of LRP6 lies in promoting canonical Wnt activation (35). In the present study, the gene expression levels of LRP6 and $\beta$-catenin were significantly reduced in the $\mathrm{M}+\mathrm{S}, \mathrm{M}+\mathrm{S}+\mathrm{OS}$ and $\mathrm{S}$ groups, whereas they were increased in the $\mathrm{M}+\mathrm{GS}, \mathrm{M}+\mathrm{GS}+\mathrm{OS}, \mathrm{GS}$ and $\mathrm{M}+\mathrm{OS}$ groups.

In K562 cells, overexpression of Wnt5a through Ror2 is absorbed by the body to activate the nonclassical Wnt5a/ Ror2/Frz4 pathway and inhibits the canonical Wnt signaling pathway. However, when Ror2 receptor expression is reduced or absent, the Wnt5a/Frz4/LRP5 classical pathway is activated (36). The objective of the present study was to test the hypothesis that LRP6 and Ror2 have an integral role in Wnt signaling in the differentiation of MSCs in calcification. The observations of the present study support this hypothesis and provide the first evidence of a well-defined pathway linking an LDL receptor-associated protein to the differentiation of MSCs in calcification. The Wnt pathways are capable of regulating inflammation and foam cell formation, pathological angiogenesis and calcification, which have critical roles in plaque formation and stability. A greater understanding of the Wnt pathways in the differentiation of MSCs in calcification may reveal novel therapeutic targets for vascular disease. The contribution of the Wnt signaling pathway in the differentiation of MSCs in calcification was investigated, indicating that specific targeting of this pathway may lead to treatment options for arteriosclerosis. In the present study, the mRNA levels of Ror2 and JNK were markedly reduced in the M+GS, $\mathrm{M}+\mathrm{GS}+\mathrm{OS}, \mathrm{GS}$ and M+OS groups only, whereas they were increased in the $\mathrm{M}+\mathrm{S}, \mathrm{M}+\mathrm{S}+\mathrm{OS}, \mathrm{S}$ and $\mathrm{M}$ groups. Although a decrease in the expression of Ror2 and JNK was also apparent in the M+GS, M+GS+OS, GS and M+OS groups, it did not reach a statistically significant difference.

However, a non-canonical Wnt-signaling pathway, known as Wnt5a/Ror2, which in general transduces through the $\mathrm{JNK} /$ planar cell polarity or the calcium-releasing pathways and regulates cell movement, can inhibit canonical Wnt-signaling (37). Wnt5a, a member of the Wnt family that is indicated to have a role in hydrophobic cell-cell interactions, is predominantly characterized as a non-canonical Wnt ligand, which activates intracellular signaling via distinct receptors or co-receptors (29). Of note, the present study revealed that Wnt5a may be involved in the pathogenesis of atherosclerosis rather than a protection from it, and the expression of Wnt5a mRNA correlates with the severity of atherosclerotic lesions (38). The present study identified that Wn5a was 
highest in the M+GS, M+GS+OS, GS and M+OS groups, but not in the $\mathrm{M}+\mathrm{S}$ and $\mathrm{S}$ groups.

Ror2, an orphan tyrosine kinase possessing an extracellular cysteine-rich Wnt binding domain (39), has been demonstrated to function as a receptor for Wnt5a (30), inducing a non-canonical cascade involving the activation of JNK and the inhibition of canonical signaling (34). The interaction between Ror2 and Wnt5a to mediate the non-canonical Wnt signaling pathway has received great attention in recent years (34). In addition, developmental phenotypes lacking Ror2 and Wnt5a lead to dwarfism, shortened limbs, facial abnormalities, ventricular septal defects in the heart and abnormalities in lung development (34). The absence of Ror2 leads to enhanced Wnt/ $\beta$-catenin signaling, specifically in cells that have lost Ror2 expression (38). This observation indicates that the intracellular domain of Ror2 is required for Wnt5a/Ror2 signaling to be functional. Overall, previous studies have indicated that Wnt5a/Ror2 signaling is capable of inhibiting canonical Wnt signaling in vivo, and point to Ror2 as being a potential therapeutic target for the treatment of human diseases. It was revealed that the mRNA levels of ROR2 and JNK were markedly reduced in the $\mathrm{M}+\mathrm{GS}, \mathrm{M}+\mathrm{GS}+\mathrm{OS}, \mathrm{GS}$ and $\mathrm{M}+\mathrm{OS}$ groups only, whereas they were increased in the $\mathrm{M}+\mathrm{S}, \mathrm{M}+\mathrm{S}+\mathrm{OS}, \mathrm{S}$ and $\mathrm{M}$ groups.

The present study provided direct evidence for a significant role of Wnt signaling mediating the differentiation of MSCs in calcification. To date, further studies remain to be undertaken in order to understand either the function of VSMCs or the complexities of the signaling pathways activated as a consequence of their stimulation or inhibition. It is expected that further studies lead to an improved comprehension of processes and substances involved in vascular remodeling and repair.

In conclusion, the present study demonstrated the expression of Wnts in the progress of differentiation of MSCs in calcification. MSCs are able to differentiate into different cell phenotypes when in direct cell-cell contact with SMCs or calcified SMCs, and the Wnt5a/Ror2 signaling pathway may be associated with the determination of the differentiation of MSCs in this process. This observation opens an avenue for the development of novel strategies of MSC transplantation for treating arteriosclerosis.

\section{References}

1. Boström KI, Rajamannan NM and Towler DA: The regulation of valvular and vascular sclerosis by osteogenic morphogens. Circ Res 109: 564-577, 2011.

2. Olivares-Navarrete R, Hyzy SL, Park JH, et al: Mediation of osteogenic differentiation of human mesenchymal stem cells on titanium surfaces by a Wnt-integrin feedback loop. Biomaterials 32: 6399-6411, 2011.

3. Abedin M, Tintut Y and Demer LL: Mesenchymal stem cells and the artery wall. Circ Res 95: 671-676, 2004.

4. Leroux L, Descamps B, Tojais NF, et al: Hypoxia preconditioned mesenchymal stem cells improve vascular and skeletal muscle fiber regeneration after ischemia through a Wnt4-dependent pathway. Mol Ther 18: 1545-1552, 2010.

5. Alfaro MP, Pagni M, Vincent A, et al: The Wnt modulator $\mathrm{SFRP}_{2}$ enhances mesenchymal stem cell engraftment, granulation tissue formation and myocardial repair. Proc Natl Acad Sci USA 105: 18366-18371, 2008.

6. Moioli EK, Clark PA, Chen M, et al: Synergistic actions of hematopoietic and mesenchymal stem/progenitor cells in vascularizing bioengineered tissues. PLoS One 3: e3922, 2008.
7. Qian H, Yang Y, Li J, et al: The role of vascular stem cells in atherogenesis and post-angioplasty restenosis. Ageing Res Rev 6: 109-127, 2007.

8. Bell GI, Meschino MT, Hughes-Large JM, et al: Combinatorial human progenitor cell transplantation optimizes islet regeneration through secretion of paracrine factors. Stem Cells Dev 21: 1863-1876, 2012.

9. Huang Z, Ren PG, Ma T, Smith RL and Goodman SB: Modulating osteogenesis of mesenchymal stem cells by modifying growth factor availability. Cytokine 51: 305-310, 2010.

10. Pelttari K, Winter A, Steck E, et al: Premature induction of hypertrophy during in vitro chondrogenesis of human mesenchymal stem cells correlates with calcification and vascular invasion after ectopic transplantation in SCID mice. Arthritis Rheum 54: 3254-3266, 2006.

11. Kramann R, Kunter U, Brandenburg VM, et al: Osteogenesis of heterotopically transplanted mesenchymal stromal cells in rat models of chronic kidney disease. J Bone Miner Res 28: 2523-2534, 2013.

12. Shabbir A, Zisa D, Suzuki G, Lee T: Heart failure therapy mediated by the trophic activities of bone marrow mesenchymal stem cells: a noninvasive therapeutic regimen. Am J Physiol Heart Circ Physiol 296: H1888-H1897, 2009.

13. Tran TC, Kimura K, Nagano M, et al: Identification of human placenta-derived mesenchymal stem cells involved in re-endothelialization. J Cell Physiol 226: 224-235, 2011.

14. Chow K, Fessel JP, Kaoriihida S, et al: Dysfunctional resident lung mesenchymal stem cells contribute to pulmonary microvascular remodeling. Pulm Circ 3: 31-49, 2013.

15. Tsaousi A, Mill C and George SJ: The Wnt pathways in vascular disease: lessons from vascular development. Curr Opin Lipidol 22: 350-357, 2011.

16. Marinou K, Christodoulides C, Antoniades C and Koutsilieris M: Wnt signaling in cardiovascular physiology. Trends Endocrinol Metab 23: 628-636, 2012.

17. Cheng CW, Yeh JC, Fan TP, et al: Wnt5a-mediated non-canonical Wnt signalling regulates human endothelial cell proliferation and migration. Biochem Biophy Res Commun 365: 285-290, 2008.

18. Ling L, Nurcombe V and Cool SM: Wnt signaling controls the fate of mesenchymal stem cells. Gene 433: 1-7, 2009.

19. Stolzing A, Jones E, McGonagle D and Scutt A: Age-related changes in human bone marrow-derived mesenchymal stem cells: consequences for cell therapies. Mech Ageing Dev 129: 163-173, 2008

20. Xin H, Xin F, Zhou S and Guan S: The Wnt5a/Ror2 pathway is associated with determination of the differentiation fate of bone marrow mesenchymal stem cells in vascular calcification. Int J Mol Med 31: 583-588, 2013.

21. Laumanns IP, Fink L, Wilhelm J, et al: The noncanonical WNT pathway is operative in idiopathic pulmonary arterial hypertension. Am J Respir Cell Mol Biol 40: 683-691, 2009.

22. Pal SN, Clancy P and Golledge J: Circulating concentrations of stem-cell-mobilizing cytokines are associated with levels of osteoprogenitor cells and aortic calcification severity. Circ J 75: 1227-1234, 2011.

23. Ball SG, Shuttleworth AC and Kielty CM: Direct cell contact influences bone marrow mesenchymal stem cell fate. Int $J$ Biochem Cell Biol 36: 714-727, 2004.

24. Yip CY and Simmons CA: The aortic valve microenvironment and its role in calcific aortic valve disease. Cardiovasc Pathol 20: 177-182, 2011.

25. Wang T, Xu Z, Jiang W and Ma A: Cell-to-cell contact induces mesenchymal stem cell to differentiate into cardiomyocyte and smooth muscle cell. Int J Cardiol 109: 74-81, 2006.

26. Mangan SH, Van Campenhout A, Rush C and Golledge J: Osteoprotegerin upregulates endothelial cell adhesion molecule response to tumor necrosis factor-alpha associated with induction of angiopoietin-2. Cardiovasc Res 76: 494-505, 2007.

27. Menge T, Gerber M, Wataha K, et al: Human mesenchymal stem cells inhibit endothelial proliferation and angiogenesis via cell-cell contact through modulation of the VE-Cadherin/ $\beta$-catenin signaling pathway. Stem Cells Dev 22: 148-157, 2013.

28. Towler DA and Demer LL: Thematic series on the pathobiology of vascular calcification: an introduction. Circ Res 108: 1378-1380, 2011.

29. Johnson ML and Rajamannan N: Diseases of Wnt signaling. Rev Endocr Metab Disord 7: 41-49, 2006. 
30. Ermakov S, Trofimov S, Malkin I and Livshits G: A significant association exists between receptor tyrosine kinase-like orphan receptor 2 gene variants and the OPG/RANKL ratio in human plasma. Osteoporos Int 23: 1899-1907, 2012.

31. Qiu W, Chen L and Kassem M: Activation of non-canonical Wnt/JNK pathway by Wnt3a is associated with differentiation fate determination of human bone marrow stromal (mesenchymal) stem cells. Biochem Biophys Res Commun 413: 98-104, 2011.

32. Tian Y, Cohen ED and Morrisey EE: The importance of Wnt signaling in cardiovascular development. Pediatr Cardiol 31: 342-348, 2010.

33. Takahashi N, Maeda K, Ishihara A, Uehara S and Kobayashi Y: Regulatory mechanism of osteoclastogenesis by RANKL and Wnt signaling. Front Biosci (Landmark Ed) 16: 21-30, 2011.

34. Grumolato L, Liu G, Mong P, et al: Canonical and noncanonical Wnts use a common mechanism to activate completely unrelated coreceptors. Genes Dev 24: 2517-2530, 2010.
35. Yuan Y, Niu CC, Deng G, et al: The Wnt5a/Ror2 noncanonical signaling pathway inhibits canonical Wnt signaling in K562 cells. Int J Mol Med 27: 63-69, 2011.

36. Laird DJ, Altshuler-Keylin S, Kissner MD, et al: Ror2 enhances polarity and directional migration of primordial germ cells. PLoS Genet 7: e1002428, 2011.

37. Christman MA, Goetz DJ, Dicherson E, et al: Wnt5a is expressed in murine and human atherosclerotic lesions. Am J Physiol Heart Circ Physiol 294: H2864-H2870, 2008.

38. Mikels A, Minami Y and Nusse R: Ror2 receptor requires tyrosine kinase activity to mediate Wnt5A signaling. J Biol Chem 284: 30167-30176, 2009.

39. Liu Y, Rubin B, Bodine PV and Billiard J: Wnt5a induces homodimerization and activation of Ror2 receptor tyrosine kinase. J Cell Biochem 105: 497-502, 2008 